\title{
Medida da visão e testes psicofísicos
}

\author{
Vision measurement and psychophysical tests
}

\author{
Airton Leite Kronbauer' \\ PauloSchor $^{2}$ \\ Luis Alberto Vieira de Carvalho ${ }^{3}$
}

\begin{tabular}{|l|}
\hline RESUMO \\
\hline A medida da visão é a base para o estudo e a padronização das ciências \\
visuais. A medida da acuidade visual tem grande importância tanto para \\
a pesquisa como para a prática clínica. Esta revisão bibliográfica (1) revê \\
os conceitos fundamentais para compreender o sentido visual e as \\
unidades de medida; (2) apresenta os limites fundamentais ao desempe- \\
nho visual e os princípios das medidas da aberração ocular; e (3) discute \\
métodos para medir e classificar a visão com novas tecnologias.
\end{tabular}

Descritores: Visão; Testes visuais; Acuidade visual; Óptica; Refração ocular; Percepção visual; Percepção de forma; Sensibilidades de contraste; Reconhecimento visual de modelos

\section{INTRODUÇÃOO}

O sentido da visão é um dos principais, senão o principal, meio de contato do ser humano com o universo. A partir do "ponto de vista" da função visual humana ocorreu o nascimento de várias ciências ${ }^{(1)}$. O conceito de Luz iniciou a partir da observação de Luz Visível, seguido por conceitos de matéria, energia e muitos outros. Necessitando de uma grande região anatômica cerebral humana para processamento intelectual ou para decodificação, a função visual é iniciada pelo estímulo do órgão visual (olho) por ondas eletromagnéticas entre 380 e $760 \mathrm{~nm}$ de comprimento de onda (conceitualmente Espectro Visível) e finalizada pelo ato de conscientização da presença de determinado padrão de estímulo ${ }^{(2)}$.

Para as ciências visuais, e particularmente para a Oftalmologia, padronizar e quantificar a visão é fundamental para estudar, diagnosticar e tratar as enfermidades oculares ${ }^{(1,3)}$. O estudo das medidas e das classificações faz parte da organização do entendimento e conhecimento total da humanidade. Muitas entidades de reconhecimento mundial são responsáveis pelo estudo e aperfeiçoamento da qualificação e quantificação, como o International Bureau of Weights and Measures - (BIPM) e a World Health Organization (WHO). A dificuldade de obter padrões perfeitos de quantificação e qualificação é motivo de discussões científicas anuais dessas entidades, com correções e ajustes dos conceitos básicos envolvidos, tal como o International System of Units (SI) e International Classification of Diseases (ICD) e International Classification of Functioning, Disability and Health (ICF) e outros ${ }^{(4)}$.

A visão é um dos sentidos do homo sapiens, tal como os sentidos táteis, álgicos, térmicos, cefaloálgicos, posicional, de equilíbrio, auditivo, olfativo, gustativo e outros. A definição de sentido é a consciência dos efeitos de um estímulo que excita um dos órgãos dos sentidos. Os sentidos acima citados possuem em comum o estímulo físico, a estrutura de captação apropriada, as vias de condução e o sistema de interpretação. Sabe-se por estudos anatômicos e fisiológicos que o sentido da visão segue padrão anatômico uniforme (as vias ópticas) até o córtex cerebral occipital; en- 
tretanto, os demais caminhos da conscientização visual ainda não estão totalmente esclarecidos. Os métodos atuais para examinar, qualificar e quantificar o sentido da visão são baseados atualmente em Testes Psicofísicos.

Os testes psicofísicos são compostos de um estímulo físico padrão e de uma resposta psíquica padrão. Ao longo da história diversos autores propuseram métodos, protocolos e unidades de medida para a visão. A inter-relação entre áreas da ciência física (óptica) e matemática (medida do estímulo físico) e da ciência biológica (medida da resposta biológica) é fundamental para compreensão dos resultados obtidos.

A proposta deste texto é revisar os conceitos modernos envolvidos no entendimento da visão e nos parâmetros de sua medida.

\section{VISÃO E FUNÇÕES VISUAIS}

As Funções Visuais, segundo a WHO, são funções sensoriais relacionadas à detecção da presença da luz, da forma, do tamanho e da cor dos estímulos visuais ${ }^{(4)}$.

Estão incluídas nestas funções: as funções da acuidade visual; do campo visual; da qualidade da visão; da detecção a luz e da cor, a variação da acuidade visual da visão distante e próxima, a visão monocular e a binocular e a qualidade visual da imagem. Estão também incluídas as disfunções, como a miopia, a hipermetropia, o astigmatismo, a hemianopsia, a cegueira de cores, a visão tubular, escotoma central e periférico, diplopia, cegueira noturna e dificuldades de adaptação à luz. Estão excluídas das funções visuais as funções de percepção.

As Funções de Percepção são funções mentais específicas de reconhecer e de interpretar estímulos sensoriais. Estão incluídas as funções da percepção auditiva, visual, olfatória, gustativa, tátil e visuo-espacial, além da alucinação e da ilusão.

\section{MEDIDA DA VISÃO E TESTES PSICOFÍSICOS}

Os dados históricos mais antigos encontrados sobre a medida da visão indicam que Kuechler, em 1843, um oftalmologista alemão, desenvolveu três tabelas de medida, mas seu trabalho foi esquecido quase completamente. Jaeger, em 1854, publicou em Viena uma tabela de leitura para documentar a visão, usada por muitos ainda hoje. Donders, em 1861, inventou o termo "acuidade visual" para descrever a qualidade da visão humana. Snellen ${ }^{(5)}$, em 1862, publicou sua famosa tabela baseada e definida em "optotipos". Snellen arbitrariamente definiu a "visão padrão" como a habilidade de reconhecer um de seus optotipos com tamanho angular de 5 minutos, sendo o optotipo formado por linhas de espessura e espaçamento de 1 minuto de arco. Desde Snellen, poucas melhorias na medida da acuidade visual foram feitas. Landolt, em 1888, propôs uma interessante modificação, com optotipos circulares anelados, com somente um elemento de quebra, com variação em sua orientação.

Apenas quase um século após Snellen, estudos atingiram sua proposta inicial. Sloan em 1959, Hyvärinen em 1976, Taylor em 1976, Bailey e Lovie em 1976 e os Protocolos Early Treatment Diabetic Retinopathy Study (EDTRS) em 1991 e International Council of Ophthalmology (ICO) em 2002 conseguiram consenso e padronizaram mundialmente a medida subjetiva da acuidade visual ${ }^{(6)}$. Estandardizou-se uma tabela com cinco letras em cada fileira, selecionadas por dificuldade, com espaçamento entre as letras e as fileiras iguais ao tamanho da letra. Desta maneira, o efeito aglomerado e o número dos erros que poderiam ser cometidos em cada linha transformaram o tamanho da letra a única variável entre os níveis de acuidade visual medidos.

Na oftalmologia, habitualmente medimos a visão através da Acuidade Visual Central (AV) com um estímulo padrão medido em graus/minutos que não são unidades consideradas pertencentes ao SI, que para medidas angulares recomenda radianos. Tanto a escala de Snellen como a escala Decimal medem AV com optotipos padronizados por minuto de arco e, até mesmo, a escala Logarítmica preconizada pelo ICO não é baseada no SI. Aproximadamente há 200 anos a oftalmologia mede a AV com optotipos medidos por minuto; levará algum tempo até o SI ser convencionado para tal finalidade.

A esquiascopia foi um dos primeiros métodos objetivos para a medida dos parâmetros refracionais da função visual, depois vieram a refração automatizada e ultimamente a aberrometria. Essas medidas objetivas da qualidade óptica ocular se relacionam com a função visual, mas não informam quanto o indivíduo enxerga. Da mesma forma, estudos eletrofisiológicos respondem pela presença ou não de atividade bioelétrica nas vias ópticas; todavia, não informam a precisa qualidade da visão. Portanto, apesar das novas técnicas, o teste psicofísico ainda é o melhor teste para avaliar qualidade da função visual. Entretanto, a AV é apenas a medida de um fator envolvido na função visual e não pode ser transformada em porcentagem visual. Esquece-se, por exemplo, da campimetria quando avaliamos a habilitação para dirigir veículos ${ }^{(7)}$.

Por outro lado, o maior problema ao analisar resultados de testes psicofísicos visuais é que o resultado encontrado corresponde à medida da resposta ao estímulo após ter sido processada pelo sistema nervoso central, sendo, portanto, a soma da função visual mais as funções mentais. Com isso, a função visual isolada não pode ser aferida com precisão através de testes psicofísicos ${ }^{(8)}$.

A medida da visão é a medida da capacidade de sentir, distinguir, discriminar, separar e resolver estímulos luminosos. Com base nos conceitos da física óptica ondulatória, a Luz ou o estímulo luminoso comporta-se como uma onda. Através da matemática (principalmente geometria analítica e trigonometria), podemos medir e padronizar o estímulo físico luminoso, sendo as principais medidas de uma onda o seu comprimento (metros-SI) e a sua amplitude (metros-SI). 
Para melhor entendimento, serão conceituadas as principais formas de medida da qualidade de visão com a descrição de alguns testes psicofísicos: (todas as medidas a seguir são preferencialmente avaliadas de maneira monocular).

\section{Mínimo visível}

Um feixe de luz circular uniforme e puntiforme de raios paralelos $(\mathrm{F})$ com energia (E) com área (A1) projeta-se na retina com uma área $(\mathrm{A} 2)$. $\mathrm{O}$ feixe $\mathrm{F}$ sofre fenômenos de difração (pupilar), de aberrações esférica e cromática que serão responsáveis por A2 apresentar-se como um círculo sem uniformidade (círculo de borramento). A capacidade de detecção visual de A2 depende do limiar de estímulo dos fotorreceptores. Portanto, a quantidade de energia de F dividido por A2 (E/A2) corresponde à energia limiar de estímulo de um cone [(E/A2)x área de um cone]. A quantidade necessária de energia de um estímulo luminoso para excitar a retina é modificada por diversas situações patológicas, mas não existe na clínica oftalmológica nenhum teste psicofísico comercialmente disponível.

\section{Mínimo separável}

Considerem dois ou mais feixes de luz lineares uniformes dispostos paralelamente (S1) com espessuras simétricas (L1) dos feixes iguais à espessura do espaço com ausência de luz que separa os feixes, projetam-se na retina como uma grade (S2) (ex.: cartões Teller). A capacidade de detecção visual de S2 depende da disposição e do número de fotorreceptores por área na retina. Os testes de AV com optotipos utilizam a habilidade de separar feixes de luz e distinguir contornos no máximo contraste. A AV corresponde à principal medida utilizada como parâmetro de qualidade visual na oftalmologia atual.

\section{Mínimo discriminável}

É a capacidade de discriminar dois feixes de luz lineares uniformes, dispostos lado a lado, com pequena diferença de intensidade luminosa entre eles. Ou de discriminar apenas um feixe de luz com pequena diferença de intensidade luminosa com um plano de fundo luminoso. A capacidade dos cones de discriminar pequenas variações de intensidade entre estímulos luminosos também é referida como sensibilidade ao gradiente de contraste ${ }^{(9)}$. O teste limiar macular da campimetria computadorizada e a tabela de sensibilidade ao contraste são alguns os testes psicofísicos mais comumente utilizados na oftalmologia. A curva de sensibilidade ao contraste é o resultado da relação entre o mínimo discriminável e o mínimo separável.

\section{Máxima visibilidade (máximo separável)}

Considerem-se dois feixes (f1 e f2) de luz puntiformes, sendo 11 contínuo e central e f2 intermitente e periférico, com distância variável de f1. A distância máxima da visibilidade conjunta de f1 e f2 vai mapear o campo de visão periférico, tendo como referência $\mathrm{f} 1$. A capacidade de percepção con- junta de f1 e f2 depende de peculiaridades anatômicas oculares e orbitárias e da área excitada de fotorreceptores na retina, além das vias ópticas relacionadas à transmissão do estímulo. A campimetria tem grande importância na oftalmologia e na neurologia, pois existe correlação anatômica precisa entre a área retínica estimulada, as vias ópticas e o córtex cerebral. A campimetria avalia a qualidade visual em função do campo de abrangência da visibilidade. O gráfico de mapeamento de isópteras na campimetria é o resultado da relação entre as medidas de mínimo discriminável por máxima visibilidade. Alguns chamam de Acuidade Visual Periférica o Campo Visual, entretanto este termo pode estar sujeito a interpretação confusa.

\section{Discriminação cromática}

Dois feixes de luz monocromática circular são dispostos lado a lado com igual intensidade luminosa e diferente comprimento de onda (cor). A capacidade dos cones discriminar variações de cor entre estímulos depende da absorção de luz por pigmentos retínicos. A combinação da excitação diferenciada desses pigmentos é responsável pela discriminação do espectro cromático. Existem vários testes psicofísicos para diagnóstico cromático, a maioria baseada em tabelas pseudoisocromáticas, sendo o mais popular no nosso meio o Teste de Ishihara.

Em comum, todos os testes psicofísicos possuem:

a) a padronização do estímulo físico.

b) a padronização da resposta psíquica.

c) o resultado, que é a medida do estímulo ou a medida da resposta.

\section{LIMITES DA VISÃO HUMANA}

Na proposta de medir a visão humana, existe a busca dos seus limites, mais do que a busca da medida média dos limites visuais populacionais, os limites de cada indivíduo são importantes $^{(10)}$. Existem alguns fatores limitadores de detalhes mais finos para o olho humano: óptico, retínico e neural.

Os fatores ópticos ou refracionais vêem sendo discutidos recentemente nos Arquivos Brasileiros de Oftalmologia (ABO) e em outras revistas. Sua importância reside na facilidade com que a oftalmologia pode corrigi-los com lentes corretoras e cirurgicamente. Certamente a correção dos fatores limitadores ópticos foi o primeiro tratamento oftalmológico à disposição da humanidade.

Os fatores retínicos ${ }^{(3)}$ são de natureza anatômica, histológica e fisiológica. Entre os limitadores desses fatores estão:

a) A qualidade, distribuição e uniformidade das células fotorreceptoras.

b) A captação de luz pelos pigmentos fotorreceptores e a função dos cones e bastonetes.

c) A qualidade da malha de ligação das células fotorreceptoras (2 tipos), células bipolares (10 a 12 tipos) e células ganglionares (15 a 20 tipos); alguns autores propõem até 15 
canais ou vias de informação retínica sendo transmitidos paralelamente (este sistema de funcionamento da retina é chamado de circuito simples ou vertical: cone-bipolar-ganglionar-cortex).

d) O funcionamento do sistema horizontal, lateral ou de "feedback" composto de neurônios intermediários (células horizontais (2 tipos), amácrinas (30 tipos) e interplexiformes).

e) Os mecanismos de fototransducção envolvidos na sensibilidade a luz e no tempo de adaptação luminosa.

Os fatores neurais são os processos neuroelétricos e neuroquímicos envolvidos nas vias ópticas, no córtex visual e nas funções mentais relacionadas à visão.

\section{FÍSICA ÓPTICA E AS MEDIDAS OCULARES}

Até alguns anos atrás, a oftalmologia e a refratometria falavam apenas em miopia, hipermetropia e astigmatismo. Entretanto, com o advento da cirurgia refrativa personalizada, das lentes multifocais e asféricas intra-oculares e da evolução da indústria óptica na fabricação de lentes, surgiu à possibilidade concreta da medicina oftalmológica intervir nas aberrações ópticas oculares de alta ordem. Até então, este interesse era reservado apenas à indústria, à física e à astronomia ${ }^{(11)}$.

Existe crescente tendência ao estudo da importância das aberrações ópticas na oftalmologia ${ }^{(12-13)}$. A influência precisa destas na função visual ainda não está esclarecida, mas são comuns os relatos clínicos de "glare" e ofuscamento relacionados à presença de aberrações de alta ordem.

A Física Óptica Moderna possui algumas fórmulas para avaliação do desempenho e da qualidade dos sistemas ópticos. A medida da qualidade da imagem pode ser baseada em algum critério do observador. Porém, a quantificação da qualidade da imagem é feita por técnicas numéricas. A Função de Transferência Óptica (OTF - Optical Transfer Function) é a medida quantitativa da qualidade da imagem. OTF descreve a estrutura de uma imagem em termos do contraste e da fase espacial num intervalo de freqüências. A análise de Forrier ${ }^{(14)}$ permite descrever qualquer objeto como a soma de componentes senoidais e por isso, a OTF pode ser usada para representar de forma detalhada uma imagem, relacionando-a com o objeto e com as aberrações introduzidas no sistema óptico ${ }^{(15)}$.

A representação da OTF é feita por uma função complexa, dependente da freqüência espacial $\left(\mathrm{n}_{\mathrm{x}}, \mathrm{n}_{\mathrm{y}}\right)$, relacionada com o contraste e com a fase da seguinte forma:

$$
\operatorname{OTF}\left(v_{x}, v_{y}\right)=\left|F\left(v_{x}, v_{y}\right)\right| e^{i \phi(v x, v y)}
$$

O primeiro termo, $\left|\mathrm{F}\left(\mathrm{v}_{\mathrm{x}}, \mathrm{v}_{\mathrm{y}}\right)\right|$, é a Função de Transferência de Modulação (MTF - Modulation Transfer Function) e o segundo é chamado de Função de Transferência de Fase (FTF).

OTF esclarece a aberração de um sistema óptico limitado a determinada freqüência. MTF expressa o valor da aberração em determinada parcela da fase expressa como (PTF). Em sistemas ópticos, o componente PTF da imagem não é capturado tipicamente pelo sensor. Assim, a medida importante com respeito aos sistemas de imagem é o MTF. A fase é criticamente importante para sistemas ópticos adaptáveis e sistemas holográficos.

A MTF pode ser definida como a razão do contraste da imagem pelo contraste do objeto, que é calculada matematicamente através da Transformada de Forrier da Função de Espalhamento Pontual (PSF - Point Spread Function). A PSF representa a distribuição da intensidade de luz de um ponto do objeto.

Para uma região onde $\phi=0$, a OTF pode ser representada apenas pela parte real, ou seja, pela MTF. A MTF, definida como a razão entre o contraste de uma imagem e do objeto associado a ela em uma determinada frequiência espacial, é apresentada como:

$$
\operatorname{MTF}\left(v_{x}, v_{y}\right)=\text { Гimagem } / \text { Гobjeto }
$$

onde, $\Gamma$ é o contraste de um período senoidal p (ou de uma freqüência espacial $v=1 / p)$, onde $\phi(v)$ é uma fase espacial dados por:

$$
\Gamma=\operatorname{Imax}-\operatorname{Imin} / \operatorname{Imax}+\operatorname{Imin}
$$

onde I é a intensidade de um objeto, podendo ser representada pela função:

$$
\mathrm{I}(\mathrm{x})=1+\Gamma \cos (2 \pi v+\phi(v))
$$

A MTF pode também ser apresentada em termos da PSF, $f(x, y)$ :

$$
\operatorname{MTF}\left(v_{x}, v_{y}\right)=\int_{-\infty}^{\infty} \int_{-\infty} f(x, y) e^{-i 2 \pi(v x x+v y y)} d x d y
$$

Dessa forma, o contraste para cada freqüência é dado pelo valor da MTF em cada frequiência.

Para realizar a medida da MTF de um sistema óptico, uma seqüência de padrões, formados por pares de linhas iluminadas claras e escuras, é projetada através do sistema óptico. O menor valor da largura dessas linhas que pode ser distinguido pelo sistema óptico é chamado de resolução do sistema e pode ser expresso por linhas por $\mathrm{mm}(\mathrm{lp} / \mathrm{mm})$. O inverso da quantidade de pares de linhas por milímetro é chamado poder de resolução, ou seja, se a imagem tem uma resolução de 50 $\mathrm{lp} / \mathrm{mm}$, um objeto com $0,01 \mathrm{~mm}$ pode ser resolvido pelo sistema óptico com uma "fidelidade" relacionada com o valor da modulação.

Num sistema ideal, a intensidade da parte iluminada da projeção desses padrões, deve ser equivalente a 1 e da parte escura deve ser zero. Isso indicaria o máximo de contraste na imagem. Porém, quando esses padrões são projetados por sistemas reais, cada linha do objeto aparece na imagem como uma linha borrada e a seção transversal de cada uma dessas linhas é chamada Função de Espalhamento Linear (LSF Line Spread Function) ${ }^{(16)}$.

\section{IMPORTÂNCIA DA MEDIDA DA VISÃO E DISCUSS Ão}

A medida da AV central é o método primordial de avaliação da saúde do sistema visual ${ }^{6}$. Essa medida é o principal 
auxílio diagnóstico usado na avaliação do sistema visual pelo médico geral e é o primeiro e principal auxílio diagnóstico do exame oftalmológico especializado. O instrumento para medida da AV é obrigatório em todos os consultórios oftalmológicos ${ }^{(17)}$.

O teste visual psicofísico é o principal método para avaliar qualidade e quantidade de visão. É usado para classificação de indivíduos normais, subnormais e amauróticos. Tem excelente desempenho para avaliar qualidade de vida $^{(18)}$.

$\mathrm{O}$ resultado do teste de AV é o principal indicativo para avaliar o benefício e desfecho em intervenção oftalmológica cirúrgica e farmacológica, sendo também a medida da AV um dos principais desfechos em pesquisas oftalmológicas.

$\mathrm{O}$ método de medida da $\mathrm{AV}$ é preferencialmente usado como triagem em campanhas de saúde visual ${ }^{(19)}$. A medida da visão central é um dos principais testes usados na medicina do tráfego para prevenção de acidentes e para finalidades legais ${ }^{(7)}$.

A medida da AV é um bom indicador da saúde visual populacional e de acesso ao serviço especializado de saúde. $\mathrm{O}$ acompanhamento do resultado da medida da AV é útil em saúde pública para prevenir doenças e complicações oftalmológicas como na retinopatia diabética ${ }^{(20)}$. A medida da visão é fundamental para avaliar tratamentos em política de saúde pública.

Todos os métodos de medida existentes, através de testes psicofísicos, desrespeitam o Sistema Internacional de Medidas (SI) e estão sendo aprimorados através de escala logarítmica. A discussão científica sobre os testes de medida da $\mathrm{AV}$ e as conotações empregadas vem sendo publicada por inúmeros autores ${ }^{(21-28)}$.

Na prática, todos os sistemas ópticos (biológico ou não biológicos) possuem certo grau de distorção da luz e da imagem. Estudar, classificar e quantificar a qualidade de um sistema óptico é fundamental para poder melhorá-lo. Para poder tratar o sistema óptico ocular é importante, antes de qualquer coisa, classificar e quantificar seu defeito.

Os métodos disponíveis atualmente possuem limitações em medidas de pessoas com visão melhor do que 20/20 e visão pior do que 20/200, possuindo, também, vieses de aferição (examinador e examinado) e de reprodutibilidade (teste-reteste), havendo a possibilidade de se aprimorar sensibilidade e especificidade em novos protocolos ${ }^{(21-25,29-33)}$.

Alguns dos métodos atuais baseados em optotipos dependem do conhecimento de letras e símbolos. Além da visão, a interpretação do optotipo depende da agilidade de raciocínio e é influenciada pelo conhecimento prévio e pela idade do examinado. Os métodos disponíveis baseados apenas em optotipos possuem o viés do modelo, do tipo, da forma e da sua disposição gráfica ${ }^{(34-35)}$. A aberração astigmática, em diferentes eixos, mesmo corrigida, pode levar à interpretação distinta do optotipo. Aberrações de alta ordem não são corrigidas com auxílios ópticos usuais, e provavelmente também são fatores de confusão na medida da visão com base em optotipos ${ }^{(36)}$.

Muitos métodos utilizam a projeção de optotipos. Entre as limitações desses métodos estão: deformação da imagem do optotipo (pela inclinação da projeção, falta de foco e distorções do sistema de lentes) e taxa de iluminação variável (calibração de iluminação imprecisa, imprecisão do sistema de luz e difração da luz de acordo com a distância de projeção). Outros métodos utilizam tabelas padronizadas com iluminação "front-lit" e "backlit". Entre as limitações desses métodos estão: dificuldade na precisão da distância do examinador e tabela (necessitando-se de fórmulas complicadas para corrigir as proporções medidas que são difíceis de padronizar em ambientes de variadas dimensões).

Os métodos baseados em optotipos medem a visão apenas quanto ao Mínimo Ângulo de Resolução (MAR) (mínimo separável). Outras medidas importantes com o Mínimo Visível (MV) (acuidade puntiforme), Mínimo Discriminável (MD) (intensidade de luz), Acuidade de Vernier, difração, adaptação, ofuscamento e outras são freqüentemente esquecidas ${ }^{(21,37-38)}$.

O padrão de medida atual pode ter seu resultado alterado indesejadamente por aberrações de baixa e alta ordem, podendo comprometer resultados de pesquisas científicas ${ }^{(21,32,36-37)}$. A evolução das análises computadorizadas (aberrometria) do sistema óptico facilita também a incorporação de novas abordagens de medida da qualidade de visão. Aprimorar novos métodos facilitará incorporação de dados para quantificar e qualificar a visão ${ }^{(39-40)}$.

Por mais incrível que pareça, a grande maioria dos métodos existentes usados para quantificação, nas mais diversas áreas da ciência, dependem da visão humana para estabelecer o ponto de corte; entretanto, ainda possuímos dúvidas para quantificar objetivamente a própria visão humana.

Os métodos disponíveis, em sua ampla maioria, ainda não incorporaram a evolução tecnológica. Essa incorporação é uma tendência mundial ${ }^{(29-31,33)}$ ainda em fase de pesquisa e desenvolvimento. A modernização digital e da inovação tecnológica está cada vez mais presente no cotidiano médico. $\mathrm{O}$ desenvolvimento de projetos de pesquisa brasileiros nesta área é de interesse social e econômico para o país. Temos um grupo formado por médicos, físicos, especialistas em computação, entre outros, cujo objetivo é desenvolver uma metodologia mais precisa para avaliação da função de transferência óptica subjetiva do olho humano e compará-la com a MTF objetiva medida em instrumentos "wavefront". Acreditamos que uma das conseqüências disso será o desenvolvimento de técnicas mais precisas e adequadas para exames de AV e também uma melhor compreensão da importância do processamento neural na qualidade visual.

\section{ABSTRACT}

Vision measurement is the basis for the study and standardization of visual sciences. Measurement of visual acuity has great value for research and for clinical practice. This paper (1) reviews the fundamental concepts to understand visual 
sense and the measuring units; (2) presents the fundamental limits to visual performance and the principles of aberration measurement of the eye; and (3) discusses methods for measuring and classifying vision with new technologies.

Keywords: Vision; Vision tests; Visual acuity; Optics; Refraction, ocular; Visual perception; Form perception; Contrast sensitivity; Pattern recognition, visual

\section{REFERÊNCIAS}

1. Newton I. Opticks: Or, A treatise of the reflections, refractions, inflexions and colours of light. $2^{\text {nd }}$ ed. London: 1717.

2. Green DG. Visual acuity, color vision and adaptation. In: Albert DM, Jakobiec FA, editors. Principles and practice of Ophthalmology. Philadelphia: Saunders; c1994. p.332-49.

3. Campbell FW, Green DG. Optical and retinal factors affecting visual resolution. J Physiol. 1965;181(3):576-93.

4. World Health Organization. The International Classification of Functioning, Disability and Health. Geneva: World Health Organization; c2001.

5. Snellen H. Letterproeven tot Bepaling der Gezichtsscherpte. Utrecht, Weyers. 1862 .

6. International Council of Ophthalmology. International standards: Visual standards: aspects and ranges of vision loss [Internet]. In: International Council of Ophthalmology 20 April 2002. Sydney, Australia. p.20-4. [cited 2007 Jun 21]. Available from: http://www.icoph.org/standards/visionres.html\#adopt

7. Colenbrander A, De Laey JJ. Visual standards: vision requirements for driving safety with emphasis on individual assessment Report prepared for the International Council of Ophthalmology [Internet]. In: 30 ${ }^{\text {th }}$ World Ophthalmology Congress; 2006 Feb 19-24; São Paulo, Brazil. San Francisco, CA: International Council of Ophthalmology; 2005 [cited 2007 Nov 26]. Available from: http://www.icoph.org/pdf/visionfordriving.pdf

8. Seth AK, Izhikevich E, Reeke GN, Edelman GM. Theories and measures of consciousness: an extended framework. Proc Natl Acad Sci U S A. 2006;103 (28):10799-804.

9. Pelli DG. Pixel independence: measuring spatial interactions on a CRT display. Spat Vis. 1997;10(4):443-6.

10. Schwiegerling J. Theoretical limits to visual performance. Surv Ophthalmol. 2000;45(2):139-46.

11. Marsack JD, Thibos LN, Applegate RA. Metrics of optical quality derived from wave aberrations predict visual performance. J Vis. 2004;4(4):322-8.

12. Jankov M, Mrochen M, Schor P, Chamon W, Seiler T. Frentes de ondas (wavefronts) e limites da visão humana. Parte 1: Fundamentos. Arq Bras Oftalmol. 2002;65(6):679-84.

13. Jankov M, Mrochen M, Schor P, Chamom W, Seiler T. Frentes de ondas (wavefronts) e limites da visão humana Parte 2: Aplicações. Arq Bras Oftalmol. 2002;65(6):685-90.

14. Fourier JB. Analyse des équations déterminées. Paris: 1831

15. Applegate RA, Thibos LN, Hilmantel G. Optics of aberroscopy and super vision. J Cataract Refract Surg. 2001;27(7):1093-107.

16. Carvalho EG. Desenvolvimento de um sistema óptico para retinografia e angiografia digital [tese]. São Carlos, Universidade de São Paulo; 2006.

17. Bicas HEA. Acuidade visual: medidas e notações. Arq Bras Oftalmol. 2002;65(3):375-84.
18. Nichols JJ, Twa MD, Mitchell GL. Sensitivity of the National Eye Institute Refractive Error Quality of Life instrument to refractive surgery outcomes. J Cataract Refract Surg. 2005;31(12):2313-8.

19. World Health Organization. Prevention of blindness \& deafness. Consultation on development of standards for characterization of visual loss and visual function [Internet]. Geneva: World Health Organization; 4 - 5 september 2003. (WHO/ PBL/03.91) [cited 2005 Nov 18]. Available from: http://whqlibdoc.who.int/hq/ 2003/WHO_PBL_03.91.pdf

20. Early Treatment Diabetic Retinopathy Study Design and baseline patient characteristics. ETDRS report number 7. Ophthalmology. 1991;98(5 Suppl): 741-56

21. Ferris FL $3^{\text {rd }}$, Kassoff A, Bresnick GH, Bailey I. New visual acuity charts for clinical research. Am J Ophthalmol. 1982;94(1):91-6.

22. Thibos LN, Hong X, Bradley A, Applegate RA. Accuracy and precision of objective refraction from wavefront aberrations. J Vis. 2004;4(4):329-51

23. Cheng X, Bradley A, Thibos LN. Predicting subjective judgment of best focus with objective image quality metrics. J Vis. 2004;4(4):310-21.

24. Cheng X, Thibos LN, Bradley A. Estimating visual quality from wavefront aberration measurements. J Refract Surg. 2003;19(5):579-84.

25. Howland B, Howland HC. Subjective measurement of high-order aberrations of the eye. Science. 1976;193(4253):580-2.

26. Bicas HEA. O idiotismo 20/20. Arq Bras Oftalmol. 2003;66(3):259.

27. Bicas HEA. Mensurações em ciência. Arq Bras Oftalmol. 2003;66(4):531-7.

28. Bicas HE, Matsushima EH, Silva JA. Visão e percepção visual. Arq Bras Oftalmol. 2003;66(1):5-8

29. Arippol PK, Salomão SR, Belfort Jr R. Método computadorizado para medida da acuidade visual. Arq Bras Oftalmol. 2007;69(6):907-14.

30. Beck RW, Moke PS, Turpin AH, Ferris FL 3rd, SanGiovanni JP, Johnson $\mathrm{CA}$, et al. A computerized method of visual acuity testing: adaptation of the early treatment of diabetic retinopathy study testing protocol. Am J Ophthalmol. 2003;135(2):194-205.

31. Cotter SA, Chu RH, Chandler DL, Beck RW, Holmes JM, Rice ML, et al Reliability of the electronic early treatment diabetic retinopathy study testing protocol in children 7 to $<13$ years old. Am J Ophthalmol. 2003;136(4):655-61.

32. Rosser DA, Murdoch IE, Cousens SN. The effect of optical defocus on the test-retest variability of visual acuity measurements. Invest Ophthalmol Vis Sci. 2004;45(4):1076-9.

33. Ruamviboonsuk P, Tiensuwan M, Kunawut C, Masayaanon P. Repeatability of an automated Landolt $\mathrm{C}$ test, compared with the early treatment of diabetic retinopathy study (ETDRS) chart testing. Am J Ophthalmol. 2003;136(4):662-9.

34. Ferris Fl $3^{\text {rd }}$, Freidlin V, Kassoff A, Green SB, Milton RC. Relative lette and position difficulty on visual acuity charts from the Early Treatment Diabetic Retinopathy Study. Am J Ophthalmol. 1993;116(6):735-40.

35. Hazel CA, Elliott DB. The dependency of $\log$ MAR visual acuity measurements on chart design and scoring rule. Optom Vis Sci. 2002;79(12):788-92. Comment in: Optom Vis Sci. 2003;80(7):486-7; author reply 487.

36. Tuan KM, Chernyak D, Feldman ST. Predicting patients' night vision complaints with wavefront technology. Am J Ophthalmol. 2006;141(1):1-6.

37. Bailey IL, Lovie JE. New design principles for visual acuity letter charts. Am J Optom Physiol Opt. 1976;53(11):740-5.

38. Enoch JM, Essock EA, Williams RA. Relating vernier acuity and Snellen acuity in specific clinical populations. Doc Ophthalmol. 1984;58(1):71-7.

39. Holladay JT. Visual acuity measurements. J Cataract Refract Surg. 2004;30 (2):287-90.

40. Nissman SA, Tractenberg RE, Saba CM, Douglas JC, Lustbader JM. Accuracy, repeatability, and clinical application of spherocylindrical automated refraction using time-based wavefront aberrometry measurements. Ophthalmology. 2006;113(4):577.e1-2.

Nos artigos enviados para publicação, o nome dos autores e suas affliações devem estar completos. Isso facilitará a indexação e os links com as bases de dados e o CV Lates. 